\title{
Design of Clothing Clustering Recommendation System on SOM Neural Network
}

\author{
Hao $\mathrm{Ma}^{1, \mathrm{a}}$ and Hao $\mathrm{Liu}^{2, \mathrm{~b}}$ \\ ${ }^{1}$ Information Engineering School \\ Beijing Institute of Fashion Technology \\ ${ }^{2}$ Information Engineering School \\ Beijing Institute of Fashion Technology \\ ${ }^{\mathrm{a}}$ gxymh@bift.edu.cn, ${ }^{\mathrm{b}} 52059676 @ q q . c o m$
}

Keywords: Personalized recommendation; SOM network; Clothing cluster recommendation

\begin{abstract}
The clothing recommendation system can record users' personalized clothing information and select records, complete the clothing recommendation function for users, and show great potential in solving personalized recommendation. In this paper, a personalized clothing recommendation method based on SOM neural network is proposed in this paper. This method analyzes the customer's personalized demand, seasonal factors and subjective factors, and carries out the clothing clustering recommendation according to the customers' choice. The test results show that the SOM neural network is used for the classification function of the clothing recommendation system, which has good accuracy and reliability.
\end{abstract}

\section{Introduction}

At present, the interdisciplinary development of the clothing industry and artificial intelligence, mutual promotion, the development prospect is broad. There are some intelligent prototype products in the field of clothing performance and evaluation recommendation, with simple recommendation index selection function, but in general, it is still in the initial stage of research. At present in the development of clothing recommendation system, how to dig through the customers choose the main factors of clothing, through the form of clothing, color, quality, customer's unique style and temperament, elegant culture and connotation, is a key problem to be solved in the clothing is recommended.

With the development of artificial intelligence technology, by the fusion of neural networks and machine learning technology processing, able to handle many nonlinear and fuzzy problems in real life, and make the system has the advantages of self-learning and adaptive. Introducing neural network technology the clothing recommendation system, can overcome the traditional clothing recommendation system method cannot solve the problem of data mining and personalized extraction, the development of clothing recommendation system is also a great role in promoting.

In the field of neural network, self-organizing Map (SOM) is an unsupervised neural network. The neural network has the advantages of strong feature extraction, effective learning and simple process. Compared with other neural network algorithms, it is more suitable for the development of complex and variable clothing recommendation system. Recommendation system designed in this paper the information collected the customer clothing choice, combined with seasonal factors and subjective factors, as neural network input signal, excavate the inherent characteristics of the personalized clothing choice, to choose a garment results in clustering analysis, recommend similar clothing for customers quickly, easily recommend to provide customers with satisfactory results.

\section{SOM Neural Network Model}

SOM self-organizing neural network can reveal the inherent law of things, the clustering similar things in the same feature space area, and will not be the same kind object clustering in different area of the feature space, so as to realize correct classification of things. SOM self-organizing neural network 
structure as shown in figure 1, the network consists of input layer and competition (output layer), has the ability of extracting input signal pattern characteristic, input layer to the input mode of one dimensional array, the input neurons receive network's input signal, the number according to the number of input vector is selected. The competition layer is a two-dimensional array, arranged by competing neurons in a certain way, arranged into a plane input layer, which is related to the neuron of the competition layer through the connection weight value.

SOM network in the training process through the adjustment of network parameters to achieve the purpose of training. SOM network adopts the recommendation process of simulation level analysis; In the future, the neural network accepts customer input data, and gradually gives the recommendation result of customer satisfaction through learning.

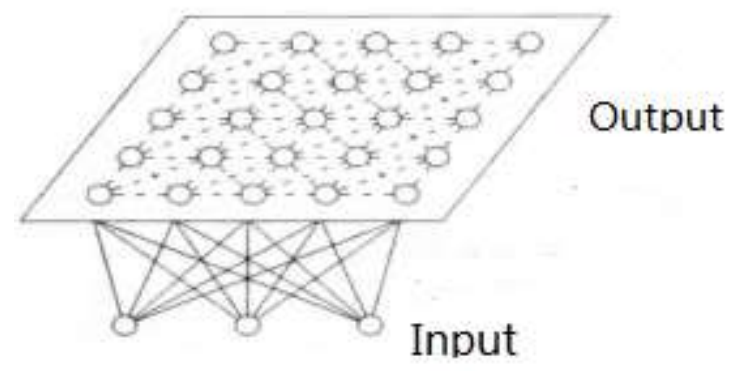

Figure 1. SOM neural network structure

The figure 1 shows that due to the topology of the SOM network as a special, so in the process of competition layer weights updated, not only of the winning neuron weight vector to get updates, and the weight vector of winning neurons neighboring neurons are tiny change of according to certain rules. In this way, with the constant adjustment of the weights between the neurons, the competition winner neurons were determined to cluster the objects according to the competing winning neurons.

To use SOM network to cluster analysis of data, we need to train and construct a SOM network. The overall process can be studied as follows:

Set a set of variables, namely input data, and standardize the input data, namely:.

Parameter initialization. The initial random set of a small set of weights, namely, and the weight of the normalized processing;

Randomly select training sample input network;

Update weight. For the winning neuron, the KOHONEN rule is adopted to customize the update of the claim weight;

Update learning rate. It is the topological domain that reclassifies the weights after learning. Adjustment of learning rate and field size is carried out in two steps: sequencing phase and adjustment stage. During training, the rate of learning decreases with time, and the adjacent area decreases with time;

Determine whether the network converges. That is, whether the training reaches the maximum value, and whether the modified amount of training is less than a certain threshold. If it does not meet the conditions, it will return 3) step. If it meets the conditions, it will end the training and output the network.

\section{Clothing Recommendation System Data Processing}

Personalized Index Data. In the clothing recommendation, there are many factors to consider, and the personalized needs of customers are the core of the clothing recommendation system. Through clothing enterprise investigation, expert consultation and questionnaire survey and analysis of the customer, combining with the characteristics of apparel and clothing personalized recommendation system of indexes can be divided into 6 types, personalized index classification as shown in table 1 .

As can be seen from table 1, skin color and body shape factor take into account the customer's own condition; The seasonal factors reflect the objective conditions; The material, color and occasion reflect 
the customer's spiritual needs. Since the expression of different customers is diverse and rich, different customers have different degrees of personalized needs.

Table1 Classification of customer personalization indicators

\begin{tabular}{|c|c|}
\hline Personalized indicator & Description \\
\hline Color of skin & White、 black \\
\hline Body type & $\begin{array}{l}\text { Female hourglass type, female inverted triangle, female triangle } \\
\text { female, oval shape, male } \mathrm{V} \text {, male straight, male pear, male fat, } \\
\text { male bulge. }\end{array}$ \\
\hline Seasons & Spring, summer, autumn and winter \\
\hline Material & $\begin{array}{l}\text { Woven fabric, cotton fabric, spandex fabric, acrylic fabric, wool } \\
\text { fabric, linen fabric, silk fabric, rayon fabric }\end{array}$ \\
\hline Occasions & $\begin{array}{l}\text { party, outdoor leisure, etc. Exercise: morning exercise, } \\
\text { after-dinner activities, etc. Business: working, meeting, meeting } \\
\text { clients, etc. }\end{array}$ \\
\hline Color & $\begin{array}{l}\text { Red, orange, yellow, green, blue, purple, black, white, grey, } \\
\text { metallic. }\end{array}$ \\
\hline
\end{tabular}

Clothing design recommendation is based on the rule of thumb in a certain, in process design rules for constraint, personalized needs in object oriented, select and match the appropriate design elements, make clothing and form a perfect harmony. Different types of clothing can meet various environmental needs, and achieve the basic cold insulation effect. Different colors can form various styles, giving people a new visual effect; Different fabrics can show their respective modeling characteristics, giving people a good spiritual enjoyment; Different design styles show the outline of various parts of the body, and the different styles are reflected through the collocation of each detail part.

Data Collection and Processing. Through the analysis of the clothing industry experts consultation and design rules from thin thickness, warm and cool colors, fabrics and design models of four elements, summarizes the design constraint rules between elements, individual characteristics and constraint rules between the various design elements, finally it puts forward the model of 25 kinds of personalized clothing clustering, the Numbers for 1 to 25.Its clustering pattern and serial number information: thin model cold color chemical fiber type 1;Light type cool cotton type 2;Thin type of cold silk and hemp loose type 3;Thin type partial cold color chemical fiber type 4; Thin and cool color cotton loose type 5; Thin and cool color silk and hemp loose type 6;Moderate type of cold color chemical fiber flat type 7;Moderate type partial cold color chemical fibre type 8;Moderate type of cold color chemical fiber sheath type 9; Moderate to cool color cotton type flat type 10;Moderate type of cool color cotton loose type 11; Moderate type of cool color cotton body type 12; Moderate type warm color chemical fiber type flat type 13;Moderate warm color chemical fibre type 14;Moderate style warm color chemical fiber type tight type 15;Moderate warm color cotton type flat 16;Moderate warm color cotton type 17;Moderate warm color cotton body type 18; Thick type of cool color cotton loose type 19; Thick style of cool color down type 20; Thick style warm color cotton loose type 21; Thick style warm color down type 22; Thick warm color cotton type loose type 23 ; Thick warm color chemical fibre type 24 ; Thick warm color down type 25.

In order to eliminate the dimensionality effect of each index, the data of each index is normalized and the SOM neural network is trained. Because of clothing recommendation system index and clustering results is more, so set the competitive layer neuron number to 48 (greater than 25 kinds of clustering) individuation, neuron learning rate is 0.001 , the distance function for LINKDIST distance, the number 
of iterations set 1000 times, in order to facilitate observation of the clustering results using compet transfer function. Save the network and use the network for cluster analysis and result analysis.

In the course of training, in view of the above recommendation system, we collected 500 characteristic samples in consideration of the final 25 clustering. In the training of characteristic samples, 400 feature samples were randomly selected as training samples, and the remaining 100 feature samples were used as test samples. Take the training sample set as input sample and enter the input layer of SOM network. Considering the structure characteristics of the output layer of SOM network, it is reasonable to define the output layer node number as (slightly larger than the clustering result).Finally, the SOM network parameter setting is shown in table 1.

To take advantage of the training sample set default SOM neural network model was trained and get SOM classification model for clothing is recommended, using test sample set for SOM classifier model test, can recommend effect validation clothing recommendation system.

\section{Analysis of Clustering Results of Personalized Clothing}

Using the training sample set by SOM self-organizing network training, the same type of costume style results in the output layer plane together and different types of clothing style on the output layer plane are separated, has realized the data feature extraction, clustering results visualization of results have been achieved.Personalized clothing system network structure as shown in figure 2, SOM network through training, will be the same kind of personalized clothing sample set gathered in the same area, there are some sample set gathered at the same point, mapped to the same winning neuron.

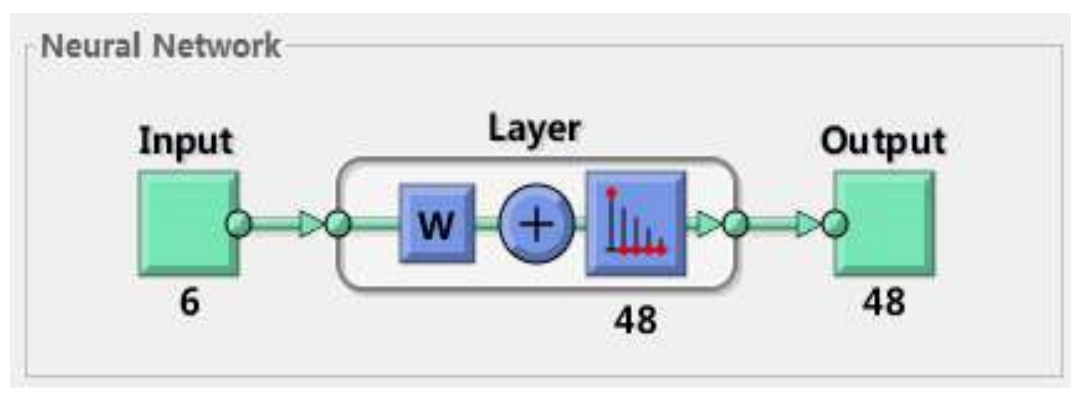

Figure2. Network structure of personalized clothing system.

In order to verify the effect of clothing recommendation system, can put the 100 test sample set in the input to the SOM network, the classification of the test sample in the output layer, test sample set in the competition results of the output layer is shown in figure 3. Test samples of the same clothing style were gathered together and mapped to the same area with the same clothing style training sample set. 


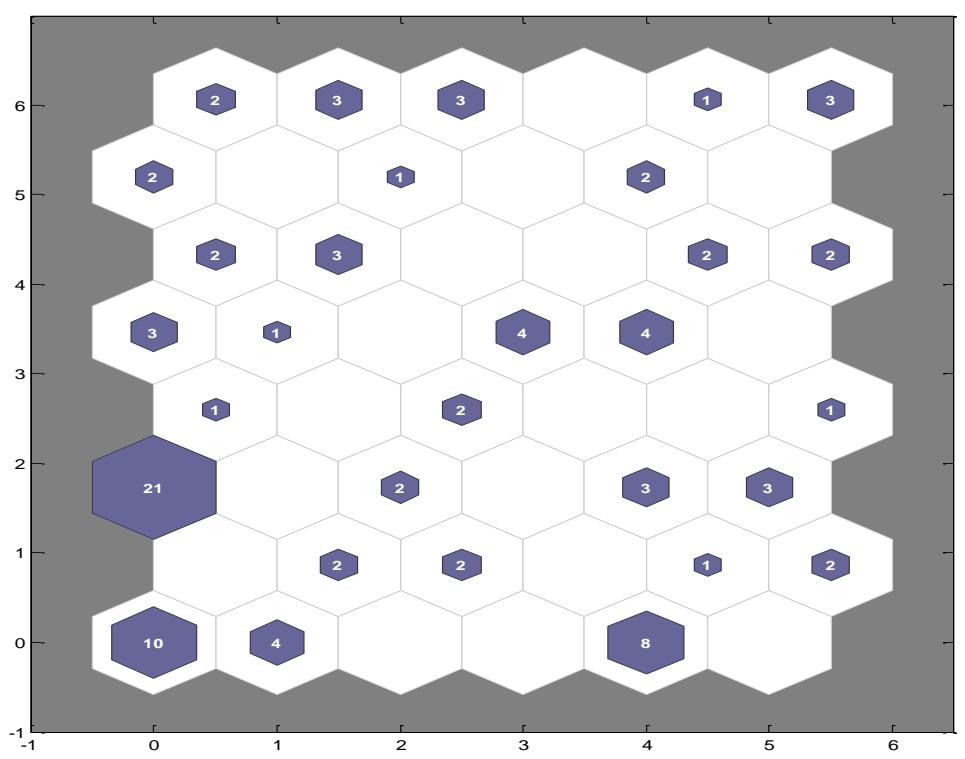

Figure 3. Test sample clustering results

\section{Conclusion}

Design method of clothing recommendation system is proposed in this paper, the method is based on the current customers personalized information is chosen as the input parameters, using SOM self-organizing network effective clustering on current personalized clothing style, so as to realize personalized recommendation of clothing. The method has good accuracy and specificity, and the recognition effect is good. However, the accuracy of the system has important relationship with the selection of the initial training sample set, which may affect the accuracy of system identification. Through the corresponding experimental analysis, SOM self-organizing network can better realize the clustering recommendation function of clothing recommendation system, which has certain engineering value.

\section{Acknowledgements}

This research was financially supported by Beijing Institute of Fashion Technology under Grant NHFZ20170060/002,NHFZ20170061/001,KYTG02170202and KYTG02170201/013.

\section{References}

[1] Yao Haini Wang Zhen, Qiu Lipeng, etc. The EMD markov distance and the application of SOM neural network in fault diagnosis research [J]. Journal of noise and vibration control, 2016, 4 (1) : 138-140162.

[2] YueYuFei Luo Jianxu. An improved application of SOM neural network fault diagnosis in sewage treatment $[\mathrm{J}]$. Journal of east China university of science and technology (natural science edition), 2017 lancet (3) : 389-396.

[3] Quan-de zhang, fruit chan, Lin Tong, etc. Based on the rolling bearing condition assessment method of self-organizing neural network [J]. China mechanical engineering, 2017, 28 (5) : $550-558$.

[4] Wang xiaoyan. Improved research and application of k-means algorithm and self-organizing neural network algorithm [D]. Taiyuan: zhongbei university, 2017.

[5] Fan guojing, tian xiuhua. A regional composite ecosystem health assessment based on SOM neural network [J]. Statistics and decision, 2017 (11) : 85-88. 